\title{
A Challenging case of Cardiac sarcoidosis diagnosed by PET scan
}

\author{
Raga Deepak Reddy Palagiri, Anusha Jillella, Fuad Habash and Hakan Paydak* \\ Department of cardiology, University of Arkansas for Medical Sciences, USA
}

\section{Introduction}

It is rare but well-known that sarcoidosis can involve the myocardium and may lead to arrhythmia or even sudden cardiac death. We present a case with atypical symptoms that led to a challenging diagnosis of cardiac sarcoidosis.

\section{Case presentation}

A 61-year-old Caucasian male with history of asthma and sleep apnea had multiple visits to the ED with dizziness. He was initially diagnosed with paroxysmal atrial fibrillation and started on metoprolol. Few weeks after discharge he came to the ED with lightheadedness and was found to have bradycardia with Mobitz type-1 heart block. So, his rate limiting medications were held and his HR has improved. He was placed on flecainide. He underwent TMST and developed complete heart block. So, flecainide was stopped. He was left on no medications as it appeared his symptoms had improved, and he didn't show any further events. This time he presented with similar complaints. His lightheadedness was sudden in onset, not related to any specific posture, no aggravating/ relieving factors but associated with diaphoresis. He didn't lose his consciousness nor sustain a fall. He is a non-smoker, occasional alcoholic and has no other drug use history. Vitals were stable including orthostatic. Pt chest x-ray showed left upper lobe infiltrate. Pt reported that he was treated for pneumonia few months ago. Nonresolution of this lesion mandated biopsy that showed non-caseating granulomas, concerning for sarcoidosis. Given his variable arrhythmia presentation with atrial fibrillation and variable AV block, there was a growing concern for myocardial involvement. Cardiac Magnetic Resonance Imaging (MRI) showed no infiltrative disease. Yet, given the high suspicion of cardiac involvement, Positron Emission Tomography (PET) scan was pursued which showed increased Fluorodeoxyglucose (FDG) uptake in interventricular septum, consistent with cardiac sarcoidosis. Pulmonary service was involved, and patient was started on prednisone and methotrexate with no future episodes of heart arrhythmias (Figure 1).

\section{Discussion}

It is rare but well-known that sarcoidosis can involve heart and cause rhythm problems and sometimes leading to SCD. His variable arrhythmias can be explained by the granulomatous involvement of the atrium and the conduction pathway. Diagnosis of cardiac sarcoidosis is often challenging given the variability in its presentation. Cardiac biopsy is not commonly done because of the patchy involvement of the

Copyright: (C2018 Raga Deepak Reddy P. This is an open-access article distributed under the terms of the Creative Commons Attribution License, which permits unrestricted use, distribution, and reproduction in any medium, provided the original author and source are credited.

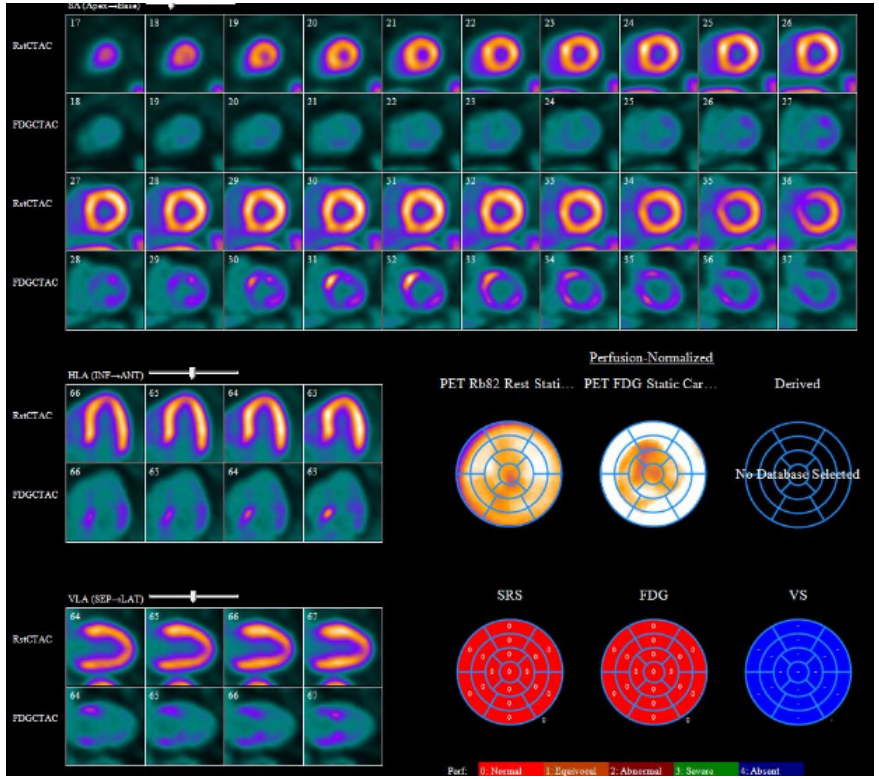

Figure 1. Cardiac sarcoidosis - PET scan

myocardium. Currently the Japanese Ministry of Health and Welfare guidelines (JMHWG) is used to diagnose cardiac sarcoidosis [1], but JMHWG incorporated cardiac MRI as a minor criterion, while PET is not included. A study was done comparing Gallium scintigraphy and PET by Rumman Langah et al, revealed that PET scan provides greater accuracy for detecting cardiac sarcoidosis; as seen in our case [2].

\section{Conclusion}

If suspicion is high for cardiac sarcoidosis, PET scan should be pursued as it has greater accuracy in detecting cardiac sarcoidosis compared to MRI.

\section{References}

1. Hiraga H (1993) "The guides for the diagnosis of cardiac sarcoidosis." Report of the Japanese Research Committee for Diffuse Lung Disease of Japan Ministry Welfare: 23-24.

2. Langah R, Spicer K, Gebregziabher M, Gordon L (2009) Effectiveness of prolonged fasting 18f-FDG PET-CT in the detection of cardiac sarcoidosis. J Nucl Cardiol 16: 801-810. [Crossref]

${ }^{\star}$ Correspondence to: Hakan Paydak, Department of cardiology, University of Arkansas for Medical Sciences, Little rock AR, 72205, USA, E-mail: Hpaydak@uams.edu

Key words: heart block, cardiac sarcoidosis, Positron emission tomography (PET) scan Received: June 06, 2018; Accepted: June 20, 2018; Published: June 25, 2018 